\title{
DENSITY ESTIMATES ON A PARABOLIC SPDE
}

\section{Márquez-Carreras ${ }^{1}$ and M. MellouK $^{2}$}

Abstract

We consider a general class of parabolic spde's

$$
\frac{\partial u_{t, x}^{\varepsilon}}{\partial t}=\frac{\partial^{2} u_{t, x}^{\varepsilon}}{\partial x^{2}}+\frac{\partial}{\partial x} g\left(u_{t, x}^{\varepsilon}\right)+f\left(u_{t, x}^{\varepsilon}\right)+\varepsilon \sigma\left(u_{t, x}^{\varepsilon}\right) \dot{W}_{t, x}
$$

with $(t, x) \in[0, T] \times[0,1]$ and $\varepsilon \dot{W}_{t, x}, \varepsilon>0$, a perturbed Gaussian space-time white noise. For $(t, x) \in(0, T] \times(0,1)$ we prove the called Davies and Varadhan-Léandre estimates of the density $p_{t, x}^{\varepsilon}$ of the solution $u_{t, x}^{\varepsilon}$.

\section{Introduction}

In this paper we deal with the perturbed parabolic stochastic partial differential equation (spde)

$$
\frac{\partial u_{t, x}^{\varepsilon}}{\partial t}=\frac{\partial^{2} u_{t, x}^{\varepsilon}}{\partial x^{2}}+\frac{\partial}{\partial x} g\left(u_{t, x}^{\varepsilon}\right)+f\left(u_{t, x}^{\varepsilon}\right)+\varepsilon \sigma\left(u_{t, x}^{\varepsilon}\right) \dot{W}_{t, x},
$$

$(t, x) \in[0, T] \times[0,1], \varepsilon>0$, with initial condition $u_{0, x}^{\varepsilon}=\xi(x)$ and Dirichlet's boundary conditions $u_{t, 0}^{\varepsilon}=u_{t, 1}^{\varepsilon}=0$. The process $\left\{\dot{W}_{t, x},(t, x) \in\right.$ $[0, T] \times[0,1]\}$ is a space-time white noise on a complete probability space $(\Omega, \mathcal{F}, P) ; \sigma, f, g: \mathbb{R} \rightarrow \mathbb{R}$ are smooth functions and $\xi$ is some real-valued function defined on $[0,1]$.

If $\sigma=f=0$ and $g(r)=r^{2} / 2$, the above equation is called Burgers equation. It arises connection with the study of turbulent fluid motion and the literature attaches great importance to this fact (see, for instance, [4]). Recently, Burgers equation perturbed by space-time white

2000 Mathematics Subject Classification. 60H15, 60H07, 35R60.

Key words. Malliavin Calculus, parabolic spde's, Davies and Varadhan-Léandre estimates, space time white noise, large deviations.

${ }^{1}$ Supported by the grant PB 960088 from the Subdirección General de Formación $y$ Promoción del Conocimiento and the grant ERBF MRX CT960075A from the European Union.

${ }^{2}$ Supported by a grant of the INRIA, domaine de Voleceau-Rocquencourt, 78153 Chesnay Cedex, France. 
noise has been considered in several papers $([\mathbf{8}],[\mathbf{9}],[\mathbf{1 2}]$ and the references therein). As $g=0,(1.1)$ is a stochastic reaction-diffusion equation, what has also been studied intensively (see, for instance, $[\mathbf{2 5}],[\mathbf{1}]$ ).

The equation (1.1) can be rigorously formulated as an integral evolution equation

$$
\begin{aligned}
& \text { 1.2) } u_{t, x}^{\varepsilon}=G_{t}(x, \xi)+\varepsilon \int_{0}^{t} \int_{0}^{1} G_{t-s}(x, y) \sigma\left(u_{s, y}^{\varepsilon}\right) W(d s, d y) \\
& -\int_{0}^{t} \int_{0}^{1} \frac{\partial G_{t-s}}{\partial y}(x, y) g\left(u_{s, y}^{\varepsilon}\right) d s d y+\int_{0}^{t} \int_{0}^{1} G_{t-s}(x, y) f\left(u_{s, y}^{\varepsilon}\right) d s d y
\end{aligned}
$$

where $G_{t}(x, y)$ is the fundamental solution of the heat equation on $[0, T] \times[0,1]$ with Dirichlet's boundary conditions and $G_{t}(x, \xi)=$ $\int_{0}^{1} G_{t}(x, y) \xi(y) d y$ (see Appendix for more information about this fundamental solution). Basic results concerning existence and uniqueness of solution of (1.1) are given in [12]. Under more restrictive assumptions on the coefficients $(f, g$ and $\sigma$ smooth enough), Morien [20] has established that, for each fixed $(t, x) \in(0, T] \times(0,1)$ and $\varepsilon \in(0,1], u_{t, x}^{\varepsilon}$ is an infinitely differentiable functional in the sense of Malliavin Calculus. Adding a strict ellipticity hypothesis, Morien has checked that $u_{t, x}^{\varepsilon}$ possesses a $C^{\infty}$ density $y \rightarrow p_{t, x}^{\varepsilon}(y)$ with respect to the Lebesgue measure. Considering the stochastic Burgers equation, i.e. $g(r)=r^{2} / 2$, and assuming a nondegeneracy condition on the diffusion coefficient, Zaidi and Nualart $[\mathbf{2 6}]$ have proved that the law of the solution is absolutely continuous. Applying techniques of Malliavin Calculus together with the Cole-Hopf transformation and assuming that the dispersion $\sigma$ does not depend on $u_{t, x}^{\varepsilon}$ and $1 / K \leq \sigma \leq K$ for some constant $K>0$, J. Léon et $a l$. [18] have shown that $u_{t, x}^{\varepsilon}$ has a smooth density at all point $(t, x) \in(0, T] \times(0,1)$.

Let $\mathcal{H}$ denote the Cameron-Martin space associated with the Brownian sheet $\left\{W_{t, x},(t, x) \in[0, T] \times[0,1]\right\}$, and set $\|h\|_{\mathcal{H}}=\left(\int_{0}^{T} \int_{0}^{1}\left|\dot{h}_{s, y}\right|^{2} d s d y\right)^{1 / 2}$, with $\dot{h}_{s, y}=\partial^{2} h_{s, y} / \partial s \partial y$. For any $h \in \mathcal{H}$, let $\left\{S_{t, x}^{h},(t, x) \in[0, T] \times[0,1]\right\}$ be the solution of the deterministic evolution equation

$$
\begin{aligned}
& S_{t, x}^{h}=G_{t}(x, \xi)+\int_{0}^{t} \int_{0}^{1} G_{t-s}(x, y) \sigma\left(S_{s, y}^{h}\right) \dot{h}_{s, y} d s d y \\
& -\int_{0}^{t} \int_{0}^{1} \frac{\partial G_{t-s}}{\partial y}(x, y) g\left(S_{s, y}^{h}\right) d s d y+\int_{0}^{t} \int_{0}^{1} G_{t-s}(x, y) f\left(S_{s, y}^{h}\right) d s d y .
\end{aligned}
$$


We set, for $y \in \mathbb{R}$,

$$
d^{2}(y)=\inf \left\{\frac{1}{2}\|h\|_{\mathcal{H}}^{2}, h \in \mathcal{H}, S_{t, x}^{h}=y\right\} .
$$

Out first aim is to prove the called Davies estimate for the density $p_{t, x}^{\varepsilon}$, which is the upper bound version of Aronson's estimates. The heat kernel case was studied by Davies [10]. Kusuoka and Stroock [14] have dealt with the diffusion processes, they have obtained a complete approach in a small time using the scaling property of the Brownian motion. For the semigroup $p_{t}(x, \cdot)$ associated with the generator of a diffusion, they obtained the upper and the lower bounds of the form $1 / \sqrt{t}$ times an exponential term related to the distance associated with the generator.

In our paper we do not have the scaling property, we will work in terms of parameter $\varepsilon$ which produces small perturbations of the solution to (1.1). Combining exponential estimates of the tail probabilities and Malliavin Calculus, we will prove that the upper bound of $p_{t, x}^{\varepsilon}(y)$ is of the form $1 / \varepsilon$ times an exponential term of the type

$$
-\frac{C\left|y-S_{t, x}^{0}\right|^{2}}{\varepsilon^{2}}
$$

for every $\varepsilon \in(0,1), y \in \mathbb{R}$, where $S_{t, x}^{0}$ is the solution to (1.3) as $h=0$. A similar result for one-dimensional wave equation perturbed by a white noise has been analysed by Léandre and Russo [17].

Secondly we analyse the logarithmic estimates for the density $p_{t, x}^{\varepsilon}$, these estimates are known as Varadhan-Léandre estimates. Assuming some conditions on the coefficients as in [20], we prove that, for fixed $(t, x)$, the density $p_{t, x}^{\varepsilon}$ decreases exponentially as $\varepsilon$ converges to 0 as follows

$$
\exp \left\{-\frac{d^{2}(y)}{\varepsilon^{2}}\right\}
$$

In the diffusion case, due to scaling property, this problem is related to the study of the density in small time. We refer to $[\mathbf{1 5}],[\mathbf{1 6}]$ for such kind of estimates. The reaction-diffusion problem, i.e. $g=0$ in (1.1), has been treated by Millet and Sanz-Solé [19].

The paper is organized as follows. In the next section we formulate the statements of the main results as Theorems 2.1 and 2.2. Section 3 is devoted to the proof of Theorem 2.1. In Section 4, we prove Theorem 2.2 and analyse the finiteness of $d^{2}(y)$ defined in (1.4). In Section 5 we apply the result of Section 3 to the reaction-diffusion equation. The arguments of Sections 3 and 4 depend on accurate estimates of the Green function $G_{t}(x, y)$, which are given as an Appendix. For all notions and 
notations concerning the Malliavin Calculus, using along the paper, we refer to $[\mathbf{2 1}],[\mathbf{2 2}]$. As usual, all constants are denoted by $C$, independently of their values.

\section{Statement of the main results}

This section is devoted to enunciate the main results of the article.

We introduce the following hypothesis on the coefficients and the initial condition:

(H1) $f, g, \sigma: \mathbb{R} \rightarrow \mathbb{R}$ and $\mathcal{C}^{\infty}$-functions with bounded derivatives of any order greater than one, $\sigma$ is uniformly bounded and $\xi \in \mathcal{C}([0,1])$.

(H2) There exists $C>0$ such that $\inf \{|\sigma(x)| ; x \in \mathbb{R}\} \geq C$.

Along the paper we fix $t \in(0, T]$ and $x \in(0,1)$.

Theorem 2.1 (Davies estimate). Assume (H1) and (H2). Then, there exist some constants $C_{1}, C_{2}>0$ such that

$$
p_{t, x}^{\varepsilon}(y) \leq \frac{C_{1}}{\varepsilon} \exp \left\{-\frac{\left|y-S_{t, x}^{0}\right|^{2}}{C_{2} \varepsilon^{2}}\right\},
$$

for any $y \in \mathbb{R}$ and $\varepsilon \in(0,1)$.

Remark. Although we assume (H1) in order to obtain Theorem 2.1, the proof still goes through under weaker conditions.

Theorem 2.2 (Varadhan-Léandre estimate). Under (H1) and (H2),

$$
\lim _{\varepsilon \downarrow 0} \varepsilon^{2} \log p_{t, x}^{\varepsilon}(y)=-d^{2}(y),
$$

with $d^{2}(y)$ defined in (1.4).

Remark. The boundedness of $\sigma$ is needed to ensure existence and smoothness of $p_{t, x}^{\varepsilon}[\mathbf{2 0}]$.

\section{Davies estimate}

In this section our main purpose is the proof of Theorem 2.1. In order to prove it we need some technical lemmas. The first one is an exponential estimate of the tail probabilities.

Lemma 3.1. Assume $f, g$ Lipschitz and $\sigma$ Lipschitz and bounded. For any $p \in[1, \infty)$, there exists $\rho>0$ large enough such that

$$
\sup _{0<\varepsilon \leq 1} E\left(\exp \left\{\frac{p\left|u_{t, x}^{\varepsilon}-S_{t, x}^{0}\right|^{2}}{\rho \varepsilon^{2}}\right\}\right)<\infty .
$$


Proof: For $(t, x) \in[0, T] \times[0,1]$, according to (1.2) and (1.3), clearly

$$
\begin{aligned}
\left|u_{t, x}^{\varepsilon}-S_{t, x}^{0}\right| \leq & \left|\int_{0}^{t} \int_{0}^{1} \frac{\partial G_{t-s}}{\partial y}(x, y)\left(g\left(u_{s, y}^{\varepsilon}\right)-g\left(S_{s, y}^{0}\right)\right) d s d y\right| \\
& +\left|\int_{0}^{t} \int_{0}^{1} G_{t-s}(x, y)\left(f\left(u_{s, y}^{\varepsilon}\right)-f\left(S_{s, y}^{0}\right)\right) d s d y\right| \\
& +\varepsilon\left|\int_{0}^{t} \int_{0}^{1} G_{t-s}(x, y) \sigma\left(u_{s, y}^{\varepsilon}\right) W(d s, d y)\right| .
\end{aligned}
$$

Hence, Lipschitz's conditions on $f$ and $g$, Schwarz's inequality, (6.1) and (6.2) yield the existence of a constant $C>0$ such that

$$
\begin{aligned}
\left|u_{t, x}^{\varepsilon}-S_{t, x}^{0}\right|^{2} \leq \varepsilon^{2}\left|\int_{0}^{t} \int_{0}^{1} G_{t-s}(x, y) \sigma\left(u_{s, y}^{\varepsilon}\right) W(d s, d y)\right|^{2} & \\
& +C \int_{0}^{t} \frac{1}{\sqrt{t-s}} \sup _{0 \leq y \leq 1}\left|u_{s, y}^{\varepsilon}-S_{s, y}^{0}\right|^{2} d s .
\end{aligned}
$$

Using Gronwall's Lemma, we obtain

$$
\sup _{0 \leq t \leq T} \sup _{0 \leq x \leq 1}\left|u_{t, x}^{\varepsilon}-S_{t, x}^{0}\right|^{2} \leq C \varepsilon^{2}\left\|\int_{0}^{\cdot} \int_{0}^{1} G \cdot-s(*, y) \sigma\left(u_{s, y}^{\varepsilon}\right) W(d s, d y)\right\|_{\infty}^{2} .
$$

Therefore, since $\sigma$ is uniformly bounded, an exponential inequality for stochastic integrals involving the Green kernel $G_{t}(x, y)$ (see Lemma 3.2 in [23] or also [24]) implies that there exist some positive constants $r_{0}$ and $C_{0}$, such that

$$
\begin{aligned}
& P\left\{\frac{\left|u_{t, x}^{\varepsilon}-S_{t, x}^{0}\right|^{2}}{\varepsilon^{2}}>r\right\} \\
\leq & P\left\{\left\|\int_{0}^{\cdot} \int_{0}^{1} G \cdot-s(*, y) \sigma\left(u_{s, y}^{\varepsilon}\right) W(d s, d y)\right\|_{\infty}^{2}>\frac{r}{C}\right\} \leq \exp \left\{-\frac{r}{C_{0}}\right\},
\end{aligned}
$$

for any $r \geq r_{0}$. 
Now, let $r_{0}, C_{0}>0$ be as before and choose $\rho>0$ large enough such that $C_{0} p<\rho$. Then, Fubini's stochastic theorem and the suitable choice of $\rho$ give

$$
\begin{aligned}
E\left(\exp \left\{\frac{p\left|u_{t, x}^{\varepsilon}-S_{t, x}^{0}\right|^{2}}{\rho \varepsilon^{2}}\right\}\right) & \leq e^{p r_{0} / \rho}+E\left(\int_{r_{0}}^{\frac{1}{\varepsilon^{2}}\left|u_{t, x}^{\varepsilon}-S_{t, x}^{0}\right|^{2}} \frac{p}{\rho} e^{\frac{p}{\rho} y} d y\right) \\
& \leq e^{p r_{0} / \rho}+\int_{r_{0}}^{\infty} \frac{p}{\rho} e^{\left(\frac{p}{\rho}-\frac{1}{C_{0}}\right) y} d y \\
& <+\infty .
\end{aligned}
$$

This concludes the proof of the lemma.

For any $\varepsilon \in(0,1)$, we consider the random variable defined by

$$
\hat{u}_{t, x}^{\varepsilon}=\frac{u_{t, x}^{\varepsilon}-S_{t, x}^{0}}{\varepsilon} .
$$

Assume (H1). Standard arguments based on Burkholder's, Hölder's and Gronwall's inequalities (see [20, Proposition 5.1]) yield for any $k \in \mathbb{N}$, $p \geq 1$,

$$
\begin{aligned}
& \sup _{0<\varepsilon<1} \sup _{t, x}\left\|u_{t, x}^{\varepsilon}\right\|_{k, p} \leq C, \\
& \sup _{0<\varepsilon<1} \sup _{t, x}\left\|\hat{u}_{t, x}^{\varepsilon}\right\|_{k, p} \leq C,
\end{aligned}
$$

where $\|\cdot\|_{k, p}$ denotes the norm of the Sobolev space $\mathbb{D}^{k, p}$, that is, for $k \in \mathbb{N}, p \geq 1$,

$$
\|F\|_{k, p}^{p}=E\left(|F|^{p}\right)+\sum_{j=1}^{k} E\left(\left\|D^{j} F\right\|_{\mathcal{H}^{j}}^{p}\right)
$$

(see $[\mathbf{2 2}]$ for basic definitions).

It only remains to study the Malliavin matrix $\gamma_{t, x}^{\varepsilon}$ of $u_{t, x}^{\varepsilon}$.

Lemma 3.2. Assume (H1) and (H2). For any $p \geq 1, \varepsilon \in(0,1)$,

$$
\left\|\left(\gamma_{t, x}^{\varepsilon}\right)^{-1}\right\|_{p} \leq C \varepsilon^{-2}
$$

where $\gamma_{t, x}^{\varepsilon}=\int_{0}^{t} \int_{0}^{1}\left|D_{r, z} u_{t, x}^{\varepsilon}\right|^{2} d r d z$ and $\|\cdot\|_{p}$ is the $L^{p}(\Omega)$-norm. 
Proof: Let $M_{t, x}^{\varepsilon}(r, z)$ be the solution of

$$
\begin{aligned}
M_{t, x}^{\varepsilon}(r, z)=G_{t-r}(x, z) & +\varepsilon \int_{r}^{t} \int_{0}^{1} G_{t-s}(x, y) \sigma^{\prime}\left(u_{s, y}^{\varepsilon}\right) M_{s, y}^{\varepsilon}(r, z) W(d s, d y) \\
& -\int_{r}^{t} \int_{0}^{1} \frac{\partial G_{t-s}}{\partial y}(x, y) g^{\prime}\left(u_{s, y}^{\varepsilon}\right) M_{s, y}^{\varepsilon}(r, z) d s d y \\
& +\int_{r}^{t} \int_{0}^{1} G_{t-s}(x, y) f^{\prime}\left(u_{s, y}^{\varepsilon}\right) M_{s, y}^{\varepsilon}(r, z) d s d y
\end{aligned}
$$

Clearly, the Malliavin derivative of $u_{t, x}^{\varepsilon}$ is given by the following equation

$$
D_{r, z} u_{t, x}^{\varepsilon}=1_{\{r<t\}} \varepsilon \sigma\left(u_{r, z}^{\varepsilon}\right) M_{t, x}^{\varepsilon}(r, z)
$$

Hence,

$$
\gamma_{t, x}^{\varepsilon}=\varepsilon^{2} \int_{0}^{t} \int_{0}^{1} \sigma\left(u_{r, z}^{\varepsilon}\right)^{2} M_{t, x}^{\varepsilon}(r, z)^{2} d r d z
$$

Computations similar to those used to prove Proposition 5.2 in [20] show that there exists a constant $C>0$ such that

$$
\sup _{0<\varepsilon<1} E\left(\int_{0}^{t} \int_{0}^{1} \sigma^{2}\left(u_{r, z}^{\varepsilon}\right) M_{t, x}^{\varepsilon}(r, z)^{2} d r d z\right)^{-p}<C
$$

for any $p \geq 1$. Consequently, (3.3) is satisfied.

We are now ready to give the proof of Theorem 2.1.

Proof of Theorem 2.1: Let $y \in \mathbb{R}$ and $\rho>0$ large enough. By a change of variable and the stochastic integration by parts formula of Malliavin Calculus (see, for instance, Proposition 3.2.1 in [22]), if $\delta_{\{y\}}$ denotes the 
Dirac $\delta$-function at $y$, then

$$
\begin{aligned}
p_{t, x}^{\varepsilon}(y)= & E\left\{\delta_{\{y\}}\left(u_{t, x}^{\varepsilon}\right)\right\} \\
= & \exp \left\{-\frac{\left|y-S_{t, x}^{0}\right|^{2}}{\rho \varepsilon^{2}}\right\} E\left\{\delta_{\{y\}}\left(u_{t, x}^{\varepsilon}\right) \exp \left\{\frac{\left|u_{t, x}^{\varepsilon}-S_{t, x}^{0}\right|^{2}}{\rho \varepsilon^{2}}\right\}\right\} \\
(3.4)= & \frac{1}{\varepsilon} \exp \left\{-\frac{\left|y-S_{t, x}^{0}\right|^{2}}{\rho \varepsilon^{2}}\right\} E\left\{\delta_{\{0\}}\left(\hat{u}_{t, x}^{\varepsilon}\right) \exp \left\{\frac{\left|u_{t, x}^{\varepsilon}-S_{t, x}^{0}\right|^{2}}{\rho \varepsilon^{2}}\right\}\right\} \\
= & \frac{1}{\varepsilon} \exp \left\{-\frac{\left|y-S_{t, x}^{0}\right|^{2}}{\rho \varepsilon^{2}}\right\} E\left\{\mathbb { 1 } _ { \{ \hat { u } _ { t , x } ^ { \varepsilon } > 0 \} } D ^ { * } \left(D \hat{u}_{t, x}^{\varepsilon}\left(\hat{\gamma}_{t, x}^{\varepsilon}\right)^{-1}\right.\right. \\
& \left.\left.\times \exp \left\{\frac{\left|u_{t, x}^{\varepsilon}-S_{t, x}^{0}\right|^{2}}{\rho \varepsilon^{2}}\right\}\right)\right\},
\end{aligned}
$$

where $\hat{\gamma}_{t, x}^{\varepsilon}=\gamma_{t, x}^{\varepsilon} / \varepsilon^{2}$ and $D^{*}$ denotes the adjoint operator of $D$, also called the Skorohod integral (see [22]).

First, notice that (3.4) is well-defined. Indeed, by Lemma 3.1, the exponential term of $(3.4)$ belongs to $\mathbb{D}_{\text {loc }}^{1, p}$ uniformly in $\varepsilon \in(0,1)$ for any $p \geq 1$ and $\rho>0$ large enough. Finally, similar arguments as in Proposition 6 in [13] (see also [2, Lemma 3.36]), together (3.2) and Lemma 3.2, yield

$$
E\left|1_{\left\{\hat{u}_{t, x}^{\varepsilon}>0\right\}} D^{*}\left(D \hat{u}_{t, x}^{\varepsilon}\left(\hat{\gamma}_{t, x}^{\varepsilon}\right)^{-1} \exp \left\{\frac{\left|u_{t, x}^{\varepsilon}-S_{t, x}^{0}\right|^{2}}{\rho \varepsilon^{2}}\right\}\right)\right|<\infty,
$$

and this completes the proof of the theorem.

Remark. As $g=0$, we deal with the well-known stochastic heat equation and $S_{t, x}^{0}$ in Theorem 2.1 is the solution to the following deterministic evolution equation

$$
v_{t, x}=G_{t}(x, \xi)+\int_{0}^{1} \int_{0}^{1} G_{t-s}(x, y) f\left(v_{s, y}\right) d s d y .
$$

\section{Varadhan-Léandre estimate}

In order to prove Theorem 2.2, we need two lemmas proved by $\mathrm{Nu}$ alart [22]. These lemmas are presented for general Wiener functionals following the formulation in the case of diffusions processes of Ben Arous's and Léandre's method (see [3]). 
Let $\{W(h), h \in \mathcal{H}\}$ be an arbitrary Gaussian family. We recall that a random variable $F: \Omega \rightarrow \mathbb{R}$ is said to be nondegenerate if $F \in \mathbb{D}^{\infty}(\mathbb{R})=$ $\bigcap_{k \geq 1} \bigcap_{p \geq 1} \mathbb{D}^{k, p}(\mathbb{R})$ and the Malliavin matrix $\gamma_{F}=\langle D F, D F\rangle_{\mathcal{H}}$ satisfies $\gamma_{F}^{-1} \in \cap_{p \geq 1} L^{p}(\Omega)$.

Lemma 4.1 ([22, Proposition 4.4.1]). Consider a family $\left\{F^{\varepsilon}, 0<\varepsilon<\right.$ 1) of nondegenerate random variables, and a function $\Phi \in \mathcal{C}_{p}^{1}(\mathcal{H}, \mathbb{R})$ such that

$$
\lim _{\varepsilon \downarrow 0} \frac{1}{\varepsilon}\left(F^{\varepsilon}\left(w+\frac{h}{\varepsilon}\right)-\Phi(h)\right)=Z(h),
$$

in the topology of $\mathbb{D}^{\infty}$, for each $h \in \mathcal{H}$, where $Z(h)$ is a random variable in the first Wiener chaos with variance $\gamma_{\Phi}(h)$. Define

$$
d_{R}^{2}(y)=\inf \left\{\frac{1}{2}\|h\|_{\mathcal{H}}^{2}, \Phi(h)=y, \gamma_{\Phi}(h)>0\right\}, \quad y \in \mathbb{R} .
$$

Then, if $p^{\varepsilon}$ denotes the density of $F^{\varepsilon}$,

$$
\liminf _{\varepsilon \downarrow 0} \varepsilon^{2} \log p^{\varepsilon}(y) \geq-d_{R}^{2}(y) .
$$

Lemma 4.2 ([22, Proposition 4.4.2]). Let $\left\{F^{\varepsilon}, \varepsilon \in(0,1)\right\}$ be a family of nondegenerate random variables satisfying

i) $\sup _{0<\varepsilon<1}\left\|F^{\varepsilon}\right\|_{k, p}<\infty$, for each $k \geq 1, p \in[1, \infty)$.

ii) For any $p \geq 1$, there exists $N(p) \in[1, \infty)$ such that $\left\|\gamma_{F^{\varepsilon}}^{-1}\right\|_{p} \leq$ $\varepsilon^{-N(p)}$ for every $\varepsilon \in(0,1]$.

iii) The family $\left\{F^{\varepsilon}, \varepsilon \in(0,1)\right\}$ satisfies a large deviation principle on $\mathbb{R}$ with rate function $I(y), y \in \mathbb{R}$.

Then, if $p^{\varepsilon}$ denotes the density of $F^{\varepsilon}$,

$$
\limsup _{\varepsilon \downarrow 0} \varepsilon^{2} \log p^{\varepsilon}(y) \leq-I(y) .
$$

We next check that $u_{t, x}^{\varepsilon}$ satisfies the requierements of Lemma 4.1 and Lemma 4.2. These two lemmas give, repectively, a lower and an upper bound of

$$
\lim _{\varepsilon \downarrow 0} \varepsilon^{2} \log p_{t, x}^{\varepsilon}(y) .
$$

Assumption (H1) implies that for fixed $(t, x) \in[0, T] \times[0,1]$, the mapping $h \in \mathcal{H} \mapsto S_{t, x}^{h}$, defined in (1.3), is infinitely Fréchet differentiable. Furthermore, the Fréchet derivative of $S_{t, x}^{h}$ is given by

$$
D S_{t, x}^{h}(k)=\int_{0}^{T} \int_{0}^{1} D_{r, z} S_{t, x}^{h} \dot{k}_{r, z} d r d z, \quad k \in \mathcal{H},
$$


with $D_{r, z} S_{t, x}^{h}=\mathbb{1}_{\{r<t\}} \sigma\left(S_{r, z}^{h}\right) \eta_{t, x}(r, z)$, and $\eta_{t, x}(r, z)$ solves the following equation

$\eta_{t, x}(r, z)=G_{t-r}(x, z)$

$$
\begin{aligned}
& +\int_{r}^{t} \int_{0}^{1} G_{t-s}(x, y)\left[\sigma^{\prime}\left(S_{s, y}^{h}\right) \dot{h}_{s, y}+f^{\prime}\left(S_{s, y}^{h}\right)\right] \eta_{s, y}(r, z) d s d y \\
& -\int_{r}^{t} \int_{0}^{1} \frac{\partial G_{t-s}}{\partial y}(x, y) g^{\prime}\left(S_{s, y}^{h}\right) \eta_{s, y}(r, z) d s d y .
\end{aligned}
$$

The proof of the following lemma is inspired by Lemma 2.5 in [19].

Lemma 4.3. Assume (H1) and (H2). Then, for any $h \in \mathcal{H}$,

$$
\gamma_{t, x}^{h}:=\int_{0}^{T} \int_{0}^{1}\left|D_{r, z} S_{t, x}^{h}\right|^{2} d r d z>0 .
$$

Remark. $\gamma_{t, x}^{h}$ is the analogue of the Malliavin matrix in the deterministic case.

Proof of Lemma 4.3: Using the strict ellipticity (H2), the proof of the lemma is reduced to check

$$
J(t, x):=\int_{0}^{t} \int_{0}^{1} \eta_{t, x}^{2}(r, z) d r d z>0 .
$$

Fix $\mu>0$ such that $0<\mu<\{t \wedge x \wedge(1-x)\}$. Then $J(t, x) \geq \frac{1}{2} J_{1}(t, x)-$ $J_{2}(t, x)$, with

$$
\begin{aligned}
& J_{1}(t, x)=\int_{t-\mu}^{t} \int_{x-\sqrt{\mu}}^{x+\sqrt{\mu}} G_{t-r}^{2}(x, z) d r d z, \\
& J_{2}(t, x)=\int_{t-\mu}^{t} \int_{x-\sqrt{\mu}}^{x+\sqrt{\mu}}\left(\eta_{t, x}(r, z)-G_{t-r}(x, z)\right)^{2} d r d z .
\end{aligned}
$$

By applying Lemma 6.1 there exist $a \geq 1, C>0$ such that, for each $0<\mu<\inf \left(t, \frac{x^{2}}{a^{2}}, \frac{(1-x)^{2}}{a^{2}}\right)$,

$$
J_{1}(t, x) \geq C \sqrt{\mu} .
$$

Let $\psi_{t}(u, x):=\int_{t-\mu}^{t} \int_{0}^{1} \eta_{u, x}^{2}(r, z) d r d z$, in order to deal with $J_{2}(t, x)$, we will prove the following

$$
\sup _{\substack{t-\mu \leq u \leq t \\ 0 \leq x \leq 1}} \psi_{t}(u, x) \leq C \sqrt{\mu} .
$$


Let $(u, x) \in[t-\mu, t] \times[0,1]$, then

$$
\psi_{t}(u, x) \leq C\left(A_{1}+A_{2}+A_{3}+A_{4}\right),
$$

with

$$
\begin{aligned}
& A_{1}=\int_{t-\mu}^{t} \int_{0}^{1} G_{u-r}^{2}(x, z) d r d z \\
& A_{2}=\int_{t-\mu}^{t} \int_{0}^{1}\left[\int_{r}^{u} \int_{0}^{1} G_{u-s}(x, y) \sigma^{\prime}\left(S_{s, y}^{h}\right) \dot{h}_{s, y} \eta_{s, y}(r, z) d s d y\right]^{2} d r d z, \\
& A_{3}=\int_{t-\mu}^{t} \int_{0}^{1}\left[\int_{r}^{u} \int_{0}^{1} G_{u-s}(x, y) f^{\prime}\left(S_{s, y}^{h}\right) \eta_{s, y}(r, z) d s d y\right]^{2} d r d z \\
& A_{4}=\int_{t-\mu}^{t} \int_{0}^{1}\left[\int_{r}^{u} \int_{0}^{1} \frac{\partial G_{u-s}}{\partial y}(x, y) g^{\prime}\left(S_{s, y}^{h}\right) \eta_{s, y}(r, z) d s d y\right]^{2} d r d z .
\end{aligned}
$$

The estimate (6.1) implies that $A_{1} \leq C \sqrt{\mu}$. Schwarz's inequality, Fubini's theorem and (6.1) imply

$$
\begin{aligned}
A_{2} & \leq C\|h\|_{\mathcal{H}}^{2} \int_{t-\mu}^{t} \int_{0}^{1} G_{u-s}^{2}(x, y)\left\{\int_{t-\mu}^{t} \int_{0}^{1} \eta_{s, y}^{2}(r, z) d r d z\right\} d s d y \\
& \leq C\|h\|_{\mathcal{H}}^{2} \int_{t-\mu}^{t} \frac{1}{\sqrt{u-s}} \sup _{0 \leq y \leq 1} \psi_{t}(s, y) d s .
\end{aligned}
$$

With less effort, $A_{3}$ can be estimated as $A_{2}$. However, the term $A_{4}$ has a special deal as consequence of the derivative of the Green kernel. Schwarz's inequality, (6.3), (6.2) and Fubini's theorem imply

$$
\begin{aligned}
A_{4} \leq & C \int_{t-\mu}^{t} \int_{0}^{1}\left[\int_{r}^{u} \int_{0}^{1}\left|\frac{\partial G_{u-s}}{\partial y}(x, y)\right| d s d y\right] \\
& \times\left[\int_{r}^{u} \int_{0}^{1}\left|\frac{\partial G_{u-s}}{\partial y}(x, y)\right| \eta_{s, y}^{2}(r, z) d s d y\right] d r d z \\
\leq & C \sqrt{\mu} \int_{t-\mu}^{t} \frac{1}{\sqrt{u-s}} \sup _{0 \leq y \leq 1} \psi_{t}(s, y) d s .
\end{aligned}
$$

Consequently, Gronwall's lemma gives (4.4). 
From (4.1) and (4.2) we obtain

$$
J_{2}(t, x) \leq C\left(B_{1}+B_{2}\right),
$$

with

$$
\begin{aligned}
B_{1}= & \int_{t-\mu}^{t} \int_{0}^{1}\left[\int _ { r } ^ { t } \int _ { 0 } ^ { 1 } G _ { t - s } ( x , y ) \left[\sigma^{\prime}\left(S_{s, y}^{h}\right) \dot{h}_{s, y}\right.\right. \\
& \left.\left.+f^{\prime}\left(S_{s, y}^{h}\right)\right] \eta_{s, y}(r, z) d s d y\right]^{2} d r d z \\
B_{2}= & \int_{t-\mu}^{t} \int_{0}^{1}\left[\int_{r}^{t} \int_{0}^{1} \frac{\partial G_{t-s}}{\partial y}(x, y) g^{\prime}\left(S_{s, y}^{h}\right) \eta_{s, y}(r, z) d s d y\right]^{2} d r d z .
\end{aligned}
$$

As before, Schwarz's inequality, Fubini's theorem, (4.4) and (6.2) yield $B_{1} \leq C \mu$. Using the same computations as $A_{4}$, we have $B_{2} \leq C \mu$. Thus,

$$
J_{2}(t, x) \leq C \mu .
$$

Hence, choose $\mu>0$ small enough, we obtain $J(t, x)>0$.

Set $U_{t, x}^{\varepsilon, h}=u_{t, x}^{\varepsilon}\left(\omega+\frac{h}{\varepsilon}\right), \varepsilon \in(0,1), h \in \mathcal{H},(t, x) \in[0, T] \times[0,1]$. The process $\left\{U_{t, x}^{\varepsilon, h},(t, x) \in[0, T] \times(0,1]\right\}$ satisfies the following evolution equation

$$
\begin{aligned}
U_{t, x}^{\varepsilon, h}=G_{t}(x, \xi) & +\varepsilon \int_{0}^{t} \int_{0}^{1} G_{t-s}(x, y) \sigma\left(U_{s, y}^{\varepsilon, h}\right) W(d s, d y) \\
& -\int_{0}^{t} \int_{0}^{1} \frac{\partial G_{t-s}}{\partial y}(x, y) g\left(U_{s, y}^{\varepsilon, h}\right) d s d y \\
& +\int_{0}^{t} \int_{0}^{1} G_{t-s}(x, y)\left\{\sigma\left(U_{s, y}^{\varepsilon, h}\right) \dot{h}_{s, y}+f\left(U_{s, y}^{\varepsilon, h}\right)\right\} d s d y
\end{aligned}
$$

By uniqueness of solution, $U_{t, x}^{0, h}=S_{t, x}^{h}$. Consider also the process $\left\{Z_{t, x}^{h},(t, x) \in[0, T] \times[0,1]\right\}$ defined by

$$
\begin{aligned}
Z_{t, x}^{h}= & \int_{0}^{t} \int_{0}^{1} G_{t-s}(x, y) \sigma\left(S_{s, y}^{h}\right) W(d s, d y)+\int_{0}^{t} \int_{0}^{1} G_{t-s}(x, y)\left\{\sigma^{\prime}\left(S_{s, y}^{h}\right) \dot{h}_{s, y}\right. \\
& \left.+f^{\prime}\left(S_{s, y}^{h}\right)\right\} Z_{s, y}^{h} d s d y-\int_{0}^{t} \int_{0}^{1} \frac{\partial G_{t-s}}{\partial y}(x, y) g^{\prime}\left(S_{s, y}^{h}\right) Z_{s, y}^{h} d s d y
\end{aligned}
$$


Notice that $Z_{t, x}^{h}$ is Gaussian. Define

$$
\hat{U}_{t, x}^{\varepsilon, h}=\frac{U_{t, x}^{\varepsilon, h}-S_{t, x}^{h}}{\varepsilon}, \quad \varepsilon \in(0,1) .
$$

Assuming (H1), one can easily check that there exists $C>0$ such that

$$
\sup _{0<\varepsilon \leq 1} \sup _{t, x} E\left(\left|U_{t, x}^{\varepsilon, h}\right|^{p}\right) \leq C, \quad \forall p \in[1, \infty) .
$$

Moreover, Gronwall's lemma and (4.5) imply

$$
\lim _{\varepsilon \downarrow 0} \sup _{x, t} E\left(\left|U_{t, x}^{\varepsilon, h}-S_{t, x}^{h}\right|^{p}\right)=0,
$$

for any $p \geq 1$.

Applying the mean-value theorem to the functions $\sigma, f, g$, and using the typical argument based on Hölder's and Burkholder-Davis-Gundy's inequalities, Gronwall's lemma and (4.6) we can ensure that

$$
L^{p}-\lim _{\varepsilon \downarrow 0}\left(\hat{U}_{t, x}^{\varepsilon, h}-Z_{t, x}^{h}\right)=0,
$$

for any $p \geq 1$, uniformly in $[0, T] \times[0,1]$.

We can also generalize the last convergence in the following way,

$$
\mathbb{D}^{\infty}-\lim _{\varepsilon \downarrow 0}\left(\hat{U}_{t, x}^{\varepsilon, h}-Z_{t, x}^{h}\right)=0 .
$$

Indeed, since $S_{t, x}^{h}$ is deterministic, $Z_{t, x}^{h}$ Gaussian and due to (4.7), in order to prove (4.8) we only need to check

$$
\begin{aligned}
& \lim _{\varepsilon \downarrow 0} E\left[\left|\int_{0}^{T} \int_{0}^{1}\left(\frac{1}{\varepsilon} D_{r, z} U_{t, x}^{\varepsilon, h}-D_{r, z} Z_{t, x}^{h}\right)^{2} d r d z\right|^{p / 2}\right]=0, \\
& \lim _{\varepsilon \downarrow 0} E\left[\left|\int_{[0, T]^{j} \times[0,1]^{j}}\left(\frac{1}{\varepsilon} D_{r, z}^{j} U_{t, x}^{\varepsilon, h}\right)^{2} d r d z\right|^{p / 2}\right]=0, \quad j=2,3, \ldots,
\end{aligned}
$$

$p \in[1, \infty)$. The validity of these two limits can be shown recursively.

Finally we state a consequence of the large deviation principle proved by Cardon-Weber [5].

Proposition 4.4. Assume (H1). Then the family of random variables $\left\{u_{t, x}^{\varepsilon}, \varepsilon \in[0,1]\right\}$ satisfies a large deviation principle with rate function $I=d^{2}$ defined in (1.4).

We can now prove Theorem 2.2. 
Proof of Theorem 2.2:

Upper bound: Let $F^{\varepsilon}=u_{t, x}^{\varepsilon}$ be the solution to (1.2). Lemma 3.2, Proposition 4.4 and the estimate (3.1) ensure that the hypothesis i)-iii) of Lemma 4.2 are fullfilled. Hence,

$$
\limsup _{\varepsilon \downarrow 0} \varepsilon^{2} \log p_{t, x}^{\varepsilon}(y) \leq-d^{2}(y) .
$$

Lower bound: Let $\Phi(h)=S_{t, x}^{h}, Z(h)=Z_{t, x}^{h}, F^{\varepsilon}=u_{t, x}^{\varepsilon}$. The assumptions of Lemma 4.1 are satisfied because of the Fréchet differentiability of $S_{t, x}^{h}$ and (4.8).

Moreover, by uniqueness of solution, $D S_{t, x}^{h}=D Z_{t, x}^{h}$. Then, Lemma 4.3 implies $d^{2}(y)=d_{R}^{2}(y)$. Consequently,

$$
\liminf _{\varepsilon \downarrow 0} \varepsilon^{2} \log p_{t, x}^{\varepsilon}(y) \geq-d^{2}(y) .
$$

Finally, we analyse the finiteness of $d^{2}(y)$ defined by (1.4). This is related to the topological support of the probabilty distribution of $u_{t, x}^{\varepsilon}$. By $[6]$

$$
\overbrace{\operatorname{supp} P \circ\left(u_{t, x}^{\varepsilon}\right)^{-1}}^{o}=\left\{z: \exists h \in \mathcal{H} \text { s.t. } S_{t, x}^{h}=z\right\} .
$$

By $[\mathbf{1 1}]$, the set $\operatorname{supp} P \circ\left(u_{t, x}^{\varepsilon}\right)^{-1}$ is a closed interval on $\mathbb{R}$. Then, we have

Proposition 4.5. Assume (H1), (H2) and the functions $f, g$, are bounded. Then

$$
\left\{z \in \mathbb{R}: d^{2}(z)<\infty\right\}=\mathbb{R} .
$$

Proof: Suppose $\sigma>\sigma_{0}>0$. Hölder's inequality, (6.1) and (6.2) imply

$$
\begin{aligned}
\left|G_{t}(x, \xi)\right| & \leq k_{1} \\
\left|\int_{0}^{t} \int_{0}^{1} G_{t-s}(x, y) f\left(S_{s, y}^{h}\right) d s d y\right| & \leq\|f\|_{\infty} k_{2} t \\
\left|\int_{0}^{t} \int_{0}^{1} \frac{\partial G_{t-s}}{\partial y}(x, y) g\left(S_{s, y}^{h}\right) d s d y\right| & \leq\|g\|_{\infty} k_{3} \sqrt{t}
\end{aligned}
$$

for some finite constants $k_{1}, k_{2}, k_{3}$. By Lemma 6.1, there exist positive constants $C$ and $\mu$ such that

$$
\int_{0}^{t} \int_{0}^{1} G_{t-s}(x, y) \sigma\left(S_{s, y}^{h}\right) d s d y \geq \sigma_{0} C \sqrt{\mu} .
$$


Let $\nu$ be a strictly positive number. For any $z \in \mathbb{R}$, define

$$
\begin{aligned}
& \dot{h}_{s, y}^{(1)}=\frac{|z|+\nu+k_{1}+k_{2} t\|f\|_{\infty}+k_{3} \sqrt{t}\|g\|_{\infty}}{C \sqrt{\mu} \sigma_{0}}, \\
& \dot{h}_{s, y}^{(2)}=-\dot{h}_{s, y}^{(1)} .
\end{aligned}
$$

One can check that

$$
S_{t, x}^{h^{(2)}}<z<S_{t, x}^{h^{(1)}}
$$

Since the topological support is a closed interval, $z \in \overbrace{\operatorname{supp} P \circ\left(u_{t, x}^{\varepsilon}\right)^{-1}}^{o}$. Then, from (4.9), there exists $\bar{h} \in \mathcal{H}$ such that $S_{t, x}^{\bar{h}}=z$.

We can use a similar argument when the coefficient $\sigma$ is negative.

Remark. Varadhan-Léandre estimate for the stochastic heat equation (i.e. $g=0$ ) have been found by Millet and Sanz-Solé [19]. In this case, we refer to [13] for more information about the set $\left\{\|h\|_{\mathcal{H}}^{2}, h \in \mathcal{H}, S_{t, x}^{h}=y\right\}$, and consequently, about $d^{2}$.

\section{Particular case: Stochastic heat equation}

Consider now the solution of the stochastic evolution equation

$$
\begin{aligned}
\bar{u}_{t, x}^{\varepsilon}=G_{t}(x, \xi)+\int_{0}^{t} \int_{0}^{1} G_{t-s}( & x, y) \sigma\left(\bar{u}_{s, y}^{\varepsilon}\right) W(d s, d y) \\
& +\int_{0}^{t} \int_{0}^{1} G_{t-s}(x, y) f\left(\bar{u}_{s, y}^{\varepsilon}\right) d s d y
\end{aligned}
$$

that means, the solution to (1.2) as $g=0$. For $h \in \mathcal{H}$, the skeleton associated with (5.1) is defined by

$$
\begin{aligned}
\psi_{t, x}^{h}=G_{t}(x, \xi)+\int_{0}^{t} \int_{0}^{1} G_{t-s}(x, y) \sigma\left(\psi_{s, y}^{h}\right) \dot{h}_{s, y} d s d y \\
\quad+\int_{0}^{t} \int_{0}^{1} G_{t-s}(x, y) f\left(\psi_{s, y}^{h}\right) d s d y
\end{aligned}
$$

Theorem 2.1 implies the following result.

Corollary 5.1. Assume (H1) and (H2). There exist some constants $C_{1}, C_{2}>0$ such that

$$
\bar{p}_{t, x}^{\varepsilon}\left(y_{0}\right) \leq \frac{C_{1}}{\varepsilon} \exp \left\{-\frac{\bar{d}^{2}\left(y_{0}\right)}{C_{2} \varepsilon^{2}}\right\}, \quad 0<\varepsilon<1 .
$$

Here $\bar{p}_{t, x}^{\varepsilon}$ denotes the density of $\bar{u}_{t, x}^{\varepsilon}$ and $\bar{d}^{2}(y)$ is the equivalent to (1.4) as $g=0$. 
Remark. In this particular case (the stochastic heat equation), for any $y_{0} \in \mathbb{R}$, we are able to find a particular element of $\mathcal{H}$ with a special structure such that applied to the skeleton is equal to $y_{0}$.

Proof of Corollary 5.1: Let $(t, x) \in[0, T] \times[0,1]$ be fixed. We will prove that, for any $y_{0} \in \mathbb{R}$, there exists $h^{(0)} \in \mathcal{H}$ satisfying $\psi_{t, x}^{h^{(0)}}=y_{0}$ and $\left\|h^{(0)}\right\|_{\mathcal{H}} \leq C\left|y_{0}-\psi_{t, x}^{0}\right|$, for some positive constant $C$ depending on $\sigma, f$ and the Green kernel. Then, $\bar{d}^{2}\left(y_{0}\right) \leq \frac{1}{2}\left\|h^{(0)}\right\|_{\mathcal{H}}^{2}$, and this fact implies (5.3).

For any $(u, z) \in[0, T] \times[0,1]$, define

$$
\begin{aligned}
\ell(u, z)=G_{u}(z, \xi)+\int_{0}^{u} \int_{0}^{1} & G_{u-s}(z, y) f\left(\psi_{s, y}^{0}\right) d s d y \\
& +\int_{0}^{u} \int_{0}^{1} G_{u-s}(z, y) \dot{k}_{t, x}(s, y) d s d y
\end{aligned}
$$

where

$$
\dot{k}_{t, x}(s, y)=G_{t-s}(x, y)\left(y_{0}-\psi_{t, x}^{0}\right)\left(\int_{0}^{t} \int_{0}^{1} G_{t-r}^{2}(x, v) d r d v\right)^{-1} .
$$

Then, $k_{t, x}(\cdot, \cdot) \in \mathcal{H}$ and it satisfies $\ell(t, x)=y_{0}$.

For any $(s, y) \in[0, T] \times[0,1]$ set

$$
\dot{h}_{s, y}^{(0)}=-\frac{f(\ell(s, y))-f\left(\psi_{s, y}^{0}\right)-\dot{k}_{t, x}(s, y)}{\sigma(\ell(s, y))} .
$$

Then, (5.4) and (5.5) imply

$$
\begin{aligned}
\ell(u, z)=G_{u}(z, \xi)+\int_{0}^{u} \int_{0}^{1} G_{u-s}(z, y) \sigma(\ell(s, y)) \dot{h}_{s, y}^{(0)} d s d y \\
\quad+\int_{0}^{u} \int_{0}^{1} G_{u-s}(z, y) f(\ell(s, y)) d s d y
\end{aligned}
$$

and, by uniqueness of solution, $\ell(u, z)=\psi_{u, z}^{h^{(0)}}$ for any $(u, z) \in[0, T] \times$ $[0,1]$. In particular $\psi_{t, x}^{h^{(0)}}=y_{0}$. Moreover, from (5.5), we have

$$
\left\|h^{(0)}\right\|_{\mathcal{H}}^{2} \leq C\left(A_{1}+A_{2}\right)
$$


with

$$
\begin{aligned}
& A_{1}=\int_{0}^{T} \int_{0}^{1}\left(\frac{f(\ell(s, y))-f\left(\psi_{s, y}^{0}\right)}{\sigma(\ell(s, y))}\right)^{2} d s d y \\
& A_{2}=\int_{0}^{T} \int_{0}^{1}\left(\frac{\dot{k}_{t, x}(s, y)}{\sigma(\ell(s, y))}\right)^{2} d s d y .
\end{aligned}
$$

Then, it is easy to check that $\left\|h^{(0)}\right\|_{\mathcal{H}}^{2} \leq C\left|y_{0}-\psi_{t, x}^{0}\right|^{2}$.

\section{Appendix}

Let $G_{t}(x, y)$ denote the fundamental solution to the heat equation with Dirichlet's boundary conditions. That means

$$
G_{t}(x, y)=\frac{1}{\sqrt{4 \pi t}} \sum_{n=-\infty}^{+\infty}\left[\exp \left\{-\frac{(y-x-2 n)^{2}}{4 t}\right\}-\exp \left\{\frac{(y+x-2 n)^{2}}{4 t}\right\}\right]
$$

We recall the following properties, for every $x, y \in[0,1], t \in[0, T], \beta>0$,

$$
G_{t}(x, y) \leq \frac{C}{\sqrt{t}} \exp \left(-\frac{(y-x)^{2}}{4 t}\right)
$$

$$
\begin{gathered}
\sup _{0 \leq x \leq 1} \int_{0}^{1}\left|G_{t}(x, y)\right|^{\beta} d y \leq C t^{-\frac{\beta}{2}+\frac{1}{2}}, \\
\sup _{0 \leq x \leq 1} \int_{0}^{1}\left|\frac{\partial G_{t}}{\partial y}(x, y)\right|^{\beta} d y \leq C t^{-\beta+\frac{1}{2}}, \\
\int_{t}^{t+h} \int_{0}^{1}\left|\frac{\partial G_{t+h-s}}{\partial y}(x, y)\right|^{\beta} d y d s \leq C_{\beta} h^{\frac{3}{2}-\beta}, \\
\text { for } h>0,0<\beta<\frac{3}{2} .
\end{gathered}
$$

We refer to [7] and [20] for the proof of results on this Green kernel.

Lemma 6.1 (Lemma 3.1 in [19]). There exists $a \geq 1$ such that for any $(t, x) \in(0, T] \times(0,1), 0<\mu<\inf \left(t, \frac{x^{2}}{a^{2}}, \frac{(1-x)^{2}}{a^{2}}\right)$,

$$
\int_{t-\mu}^{t} \int_{x-\sqrt{\mu}}^{x+\sqrt{\mu}} G_{t-s}^{2}(x, y) d s d y \geq C \sqrt{\mu}
$$

where $C=\frac{1}{4} \sqrt{\frac{2}{\pi}}\left(1-\frac{1}{\sqrt{2 \pi}}\right)$. 


\section{References}

[1] V. Bally and E. Pardoux, Malliavin calculus for white noise driven parabolic SPDEs, Potential Anal. 9(1) (1998), 27-64.

[2] G. Ben Arous, Développement asymptotique du noyau de la chaleur hypoelliptique hors du cut-locus, Ann. Sci. École Norm. Sup. (4) 21(3) (1988), 307-331.

[3] G. Ben Arous and R. LÉandre, Décroissance exponentielle du noyau de la chaleur sur la diagonale. II, Probab. Theory Related Fields 90(3) (1991), 377-402.

[4] J. M. Burgers, "The nonlinear diffusion equation", D. Reidel Publishing Company, Dordrecht, 1974.

[5] C. Cardon-Weber, Large deviations for a Burgers'-type SPDE, Stochastic Process. Appl. 84(1) (1999), 53-70.

[6] C. Cardon-Weber and A. Millet, A support theorem for a generalized Burgers SPDE, Potential Anal. 15(4) (2001), 361-408.

[7] F. Chenal and A. Millet, Uniform large deviations for parabolic SPDEs and applications, Stochastic Process. Appl. 72(2) (1997), 161-186.

[8] G. Da Prato, A. Debussche and R. Temam, Stochastic Burgers' equation, NoDEA Nonlinear Differential Equations Appl. 1(4) (1994), 389-402.

[9] G. Da Prato and D. Gatarek, Stochastic Burgers equation with correlated noise, Stochastics Stochastics Rep. 52(1-2) (1995), $29-41$.

[10] E. B. Davies, "Heat kernels and spectral theory", Cambridge Tracts in Mathematics 92, Cambridge University Press, Cambridge, 1989.

[11] S. FAng, Une inégalité isopérimétrique sur l'espace de Wiener, Bull. Sci. Math. (2) 112(3) (1988), 345-355.

[12] I. GYÖNGY, Existence and uniqueness results for semilinear stochastic partial differential equations, Stochastic Process. Appl. 73(2) (1998), 271-299.

[13] A. Kohatsu-Higa, D. Márquez-Carreras and M. SanzSolé, Asymptotic behavior of the density in a parabolic SPDE, J. Theoret. Probab. 14(2) (2001), 427-462.

[14] S. Kusuoka AND D. Stroock, Applications of the Malliavin calculus. III, J. Fac. Sci. Univ. Tokyo Sect. IA Math. 34(2) (1987), 391-442. 
[15] R. LÉAndre, Estimation en temps petit de la densité d'une diffusion hypoelliptique, C. R. Acad. Sci. Paris Sér. I Math. 301(17) (1985), 801-804.

[16] R. LÉANDRE, Intégration dans la fibre associée à une diffusion dégénérée, Probab. Theory Related Fields 76(3) (1987), 341-358.

[17] R. LÉAndre And F. Russo, Density estimates for stochastic partial differential equations, in: "Seminar on Stochastic Analysis, Random Fields and Applications" (Ascona, 1993), Progr. Probab. 36, Birkhäuser, Basel, 1995, pp. 169-186.

[18] J. A. León, D. Nualart and R. Pettersson, The stochastic Burgers equation: finite moments and smoothness of the density, Infin. Dimens. Anal. Quantum Probab. Relat. Top. 3(3) (2000), 363-385.

[19] A. Millet And M. SAnz-Solé, Varadhan estimates for the density of the solution to a parabolic stochastic partial differential equation, in: "Stochastic analysis and applications" (Powys, 1995), World Sci. Publishing, River Edge, NJ, 1996, pp. 330-342.

[20] P.-L. Morien, On the density for the solution of a Burgerstype SPDE, Ann. Inst. H. Poincaré Probab. Statist. 35(4) (1999), 459-482.

[21] D. Nualart, "The Malliavin calculus and related topics", Probability and its Applications, Springer-Verlag, New York, 1995.

[22] D. Nualart, David Analysis on Wiener space and anticipating stochastic calculus, in: "Lectures on probability theory and statistics" (Saint-Flour, 1995), Lecture Notes in Math. 1690, Springer, Berlin, 1998, pp. 123-227.

[23] C. Rovira And S. Tindel, Sharp Laplace asymptotics for a parabolic SPDE, Stochastics Stochastics Rep. 69(1-2) (2000), 11-30.

[24] R. B. Sowers, Large deviations for a reaction-diffusion equation with non-Gaussian perturbations, Ann. Probab. 20(1) (1992), $504-537$.

[25] J. B. WALsh, An introduction to stochastic partial differential equations, in: "École d'été de probabilités de Saint-Flour, $X I V-1984 "$, Lecture Notes in Math. 1180, Springer, Berlin, 1986, pp. 265-439.

[26] N. L. ZAIDI AND D. NuAlart, Burgers equation driven by a spacetime white noise: absolute continuity of the solution, Stochastics Stochastics Rep. 66(3-4) (1999), 273-292. 
Facultat de Matemàtiques

Universitat de Barcelona

Gran Via 585

08007 Barcelona

Spain

E-mail address: marquez@mat.ub.es

E-mail address: mmellouk@mat.ub.es

Primera versió rebuda el 14 de febrer de 2001, darrera versió rebuda el 18 d'octubre de 2001. 